\title{
Zebra pattern in decametric radio emission of Jupiter
}

\author{
M. Panchenko ${ }^{1}$, S. Rošker ${ }^{2}$, H. O. Rucker ${ }^{2}$, A. Brazhenko ${ }^{3}$, P. Zarka ${ }^{4,5}$, G. Litvinenko ${ }^{6}$, V. E. Shaposhnikov ${ }^{7,8}$, \\ A. A. Konovalenko ${ }^{6}$, V. Melnik ${ }^{6}$, A. V. Franzuzenko ${ }^{3}$, and J. Schiemel ${ }^{2}$ \\ 1 Space Research Institute, Austrian Academy of Sciences, Schmiedlstrasse 6, 8042 Graz, Austria \\ e-mail: Mykhaylo.Panchenko@oeaw.ac.at \\ 2 Commission for Astronomy, Austrian Academy of Sciences, Schmiedlstrasse 6, 8042 Graz, Austria \\ 3 Institute of Geophysics, Gravimetric Observatory, 36014 Poltava, Ukraine \\ ${ }^{4}$ LESIA, Observatoire de Paris, CNRS, PSL, 92195 Meudon, France \\ 5 Station de Radioastronomie de Nançay, Observatoire de Paris, CNRS, PSL, Univ. Orléans, 18330 Nançay, France \\ 6 Institute of Radio Astronomy, 61002 Kharkiv, Ukraine \\ 7 Institute of Applied Physics, 603950 Nizhny Novgorod, Russia \\ 8 National Research University High School of Economics, 603950 Nizhny Novgorod, Russia
}

Received 13 June 2017 / Accepted 6 October 2017

\begin{abstract}
We report the systematic analysis of zebra-like fine spectral structures in decametric frequency range of Jovian radio emission. Observations were performed by the large ground-based radio telescope URAN-2 during three observation campaigns between, Sep., 2012, and May, 2015. In total, 51 zebra pattern (ZP) events were detected. These rare fine radio features are observed in frequency range from 12.5 to $29.7 \mathrm{MHz}$ as quasi-harmonically related bands of enhanced brightness. ZPs are strongly polarized radio emission with a duration from $20 \mathrm{~s}$ to $290 \mathrm{~s}$ and flux densities $\sim 10^{5}-10^{6} \mathrm{Jy}$ (normalized to $1 \mathrm{AU}$ ), that is, 1-2 orders lower than for Iodecametric radio emission (DAM). Occurrence of the events does not depend on the position of Io satellite but is strongly controlled by the Jovian central meridian longitude (CML). ZPs are mainly detected in two active sectors of Jovian CMLs: $100^{\circ}$ to $160^{\circ}$ for Northern sources (right-handed polarized) and $300^{\circ}$ and $60^{\circ}\left(\right.$ via $360^{\circ}$ ) for the Southern sources (left-handed). The frequency interval between neighboring stripes is from 0.26 to $1.5 \mathrm{MHz}$ and in most cases this interval increases with frequency. We discussed the double plasma resonance with electrons or ions as a possible source of the ZPs. The performed analysis of the observations allows us to conclude that the observed ZPs are a new type of narrow band spectral structures in the Jovian DAM.
\end{abstract}

Key words. planets and satellites: individual: Jupiter - radiation mechanisms: non-thermal - radio continuum: planetary systems instabilities - waves

\section{Introduction}

Jupiter, with the largest planetary magnetosphere in the solar system, is the source of complex, powerful non-thermal radio emission in wide frequency band extended from several $\mathrm{kHz}$ up to hundreds of MHz. This emission is the result of complicated interactions between the exceptionally dynamic Jovian magnetosphere and energetic particles supplying the free energy from planetary rotation and the interaction between Jupiter and its moons (in mainly Io). Jovian decametric radio emission (DAM) is the strongest component of the radio emission of Jupiter observed in a frequency range from a few $\mathrm{MHz}$ up to $40 \mathrm{MHz}$. Frequency-time spectra exhibit a very complex structure. For example, as a result of non-axisymmetric magnetic field and non-isotropic emission patterns, DAM exhibits strong periodicity related to the rotation of the Jupiter's magnetosphere (9.92492 h; System III, 1965) and Io orbital period (42.46 h; Carr et al. 1983; Kaiser 1993). Panchenko et al. (2010) and Panchenko et al. (2013) reported on the finding of new types of intense non-Io DAM bursts which recurred very periodically during several of Jupiter's rotations, with an average period of $\sim 10.07 \mathrm{~h}$, which is $\sim 1.5 \%$ longer than the period of System III (1965). The "long" L-burst emission or wide-band noise radio storms vary at a time scale of minutes, whereas very short impulsive S-bursts are characterized by micro-milliseconds duration (Carr et al. 1983; Zarka 1998; Ryabov et al. 2014). The L-bursts are observed in the form of arc-shaped radio bursts and the S-bursts are detected as short impulsive spikes (as single events or as groups of S-burst trains) with fast drift in the time-frequency plane (Riihimaa 1977; Leblanc et al. 1980; Litvinenko et al. 2000). The S-bursts and part of the L-bursts (so-called Io-DAM) demonstrate dependence on the orbital position of the Io moon and are produced in the vicinity of Io flux tube. Independently from the Io's orbital position, part of the Lbursts (non-Io-DAM) feel a notable influence of the solar wind also through their magnetic sector structure and density fluctuations (Hess et al. 2014). This has been attributed to the high latitude of the sources of non-Io-DAM (Zarka 1998, and references therein).

Jovian decametric radiation has a rich spectral structure. The modulation effects in the dynamic spectra of S-bursts have been discussed in a large number of works (see, Riihimaa 1979; Genova et al. 1981; Imai et al. 1997; Litvinenko et al. 2009; Ryabov et al. 2014). In particular, Riihimaa (1979) discovered structures in the form of groups of parallel lanes (so-called "modulation lanes"), drifting in the time-frequency plane with positive or negative sign of drift, and, in some cases, their superposition. Recently, we reported a new fine structure of non-IoDAM in the form of quasi harmonic parallel strips of enhanced brightness drifting synchronously in time similar to the zebra patterns (ZPs) in the solar radiation (Litvinenko et al. 2016). Similar structures were observed by the RPWS (Radio and Plasma Wave Science) instrument onboard the Cassini mission 
during its Jupiter flyby in the Jovian broad-band kilometric radiation at low frequencies 30-79 kHz (Kurth et al. 2001).

In this paper we report the first systematic analysis of 51 ZP events recorded in decametric frequency range $(10-30 \mathrm{MHz})$ of Jovian DAM. The data were obtained during three observation campaigns of Jupiter in 2012-2015 by URAN-2 (Poltava, Ukraine) ground-based radio telescope. We suggest that ZPs can be considered as a new type of fine spectral structures of Jovian decametric radio emission.

In Sect. 2 we briefly describe instruments used for observation of Jovian decametric radiation. Examples of dynamic spectra with the fine structure in the form of quasi parallel strips of enhanced brightness drifting synchronously in time are presented in Sect. 3 including results of polarization observation (Sect. 3.1) and of analysis of ZPs distribution in CML and Io phase coordinates (Sect. 3.2). Statistical investigation of parameters of ZPs is performed in Sect. 3.3. In Sect. 3.4 we present the simultaneous observations of the ZPs by two radio telescopes UTR-2 and URAN-2. In the Sect. 4 we discuss possible mechanisms of ZP generation.

\section{Observations}

URAN is a decametric VLBI network which includes five telescopes: UTR-2, URAN-1, URAN-2, URAN-3, and URAN-4 (Konovalenko et al. 2016). In our study we used mainly the data from URAN-2 (Poltava, Ukraine) which is operated in the frequency range $8-32 \mathrm{MHz}$ (Brazhenko et al. 2005; Konovalenko et al. 2016). The antenna array of URAN-2 consists of 512 pairs of linearly polarized crossed dipoles (dualpolarization antennas). The signals from each individual dipole are combined into analog phased array. The effective area of the URAN-2 array is $28000 \mathrm{~m}^{2}$ with a beam pattern size of $3.5^{\circ} \times 7^{\circ}$ (at $25 \mathrm{MHz}$ ). The URAN-2 system provides full polarization capability, allowing all four Stokes parameters of the radio signal to be determined. Since 2005 URAN-2 performs regular monitoring of solar radio emmision, and since Sep., 2012, also Jovian radio emissions.

UTR-2 is a T-shape telescope located near Kharkiv in northeast Ukraine (Konovalenko 2000; Konovalenko et al. 2016) and has the world's largest antenna array operated in decameter frequency range 8-32 $\mathrm{MHz}$. Its huge collecting area consists of 2040 antenna elements in two arms North-South $(1800 \times 60 \mathrm{~m})$ and East-West $(900 \times 60 \mathrm{~m})$. Each of these arms can be operated independently in full power and cross-correlation modes providing multi-beam capability. The collective area of the UTR-2 is $140000 \mathrm{~m}^{2}$ and the angular resolution is $25 \operatorname{arcmin}$ at $25 \mathrm{MHz}$.

URAN-2 and UTR-2 are equipped with several highperformance wave-form receivers and digital spectrometers $\mathrm{DSPz}$ providing the observations with high temporal (up to microseconds) and frequency resolution (4 kHz; Ryabov et al. 2010; Zakharenko et al. 2016). The telescopes are equipped with a broadband system of antenna amplification which is very resistant to interference influences (Abranin et al. 2001). In the decametric frequency range the system temperature of radio telescopes is dominated by the Galactic background brightness temperature which is about $40-120 \mathrm{kK}$ at $20 \mathrm{MHz}$ (e.g., Konovalenko et al. 2016, Fig. 21). The sensitivity of URAN-2 and UTR-2 has been calibrated using the Galactic background radiation as a flux reference radio source (e.g., Dulk et al. 2001).

In our study we analyzed the data obtained during three observation campaigns on the radio telescope URAN-2 in Poltava, Ukraine (see Table 1). The campaigns included long-term continuous observation of Jovian radio emission (during periods of Jupiter's visibility) in the frequency range $8-32 \mathrm{MHz}$, with time-frequency resolution $0.1 \mathrm{~s} \times 4 \mathrm{kHz}$. In parallel, several short-time coordinated observational campaigns were organized on URAN-2 and UTR-2 during which both telescopes simultaneously observed Jupiter. Unique observational material was obtained including many events of Io controlled DAM, non-Io DAM as well as fine spectral structures such as narrow band events and S-bursts. In total, 579 observation sessions were performed during these three campaigns. Each of the sessions lasted from about 3 to $9 \mathrm{~h}$, depending on Jupiter's visibility above the horizon.

\section{Zebra patterns in decametric radio emission}

Over the course of the analysis of the dynamic spectra we have found a new type of fine structure in the decametric frequency range of Jovian radio emission, namely, "zebra" stripe-like spectral structures first reported by (Litvinenko et al. 2016). These ZPs are observed as quasi-harmonically related bands (stripes) of enhanced brightness drifting in parallel in time. The minimum duration of one single ZP event was $20 \mathrm{~s}$ and the maximum was $4 \mathrm{~min} 50 \mathrm{~s}$. The mean duration averaged over all zebra events was $120 \mathrm{~s}$.

Examples of dynamic spectra with the ZPs are shown in Fig. 1. In total, 51 clear individual events were detected between Sep., 2012, and May, 2015. These 51 ZP events were observed in only 42 out of all 579 observation sessions, thus ZP is a rather rare phenomenon in Jovian radio emission.

In this section we provide the statistics of the main properties of the ZPs. The results of the statistical analysis should provide the basis for the theoretical framework of the Jovian zebra phenomenon. Each zebra event is characterized by time duration, frequency range, number of stripes, frequency splitting between stripes, degree of circular polarization, as well as the central meridian longitude (CML) and Io phase of the URAN-2 at the moment of the event observation. We define the single radio event of ZPs as a spectral structure with clear onset and offset times, and with minimum three stripes, as shown in Fig. 1. Sometimes ZPs were observed as a sequence of several single zebra events, as shown in Fig. 1d. The number of stripes varies with time.

Figure 1a shows striped spectral structure recorded on 10 Nov., 2012 in the time interval 23:46:15-23:49:30 UT in the frequency range between 20 and $26.5 \mathrm{MHz}$. It is clearly seen that the number of stripes and their observational frequency vary with time. Up to five individual emission stripes were recorded simultaneously between 23:46:20-23:47:00 UT and 23:47:3023:48:00 UT. The duration of the event was $3.5 \mathrm{~min}$.

Figure $1 \mathrm{~b}$ shows the observation on 11 Nov., 2012. The most prominent four stripes were observed in the time interval between 21:07:40 and 21:08:10 UT in frequencies 12.5-13.5 MHz. The striped structures were detected during a $30 \mathrm{~s}$ period, that is, significantly shorter than that for events in Fig. 1a.

Figure $1 \mathrm{c}$ is the dynamic spectrum observed by URAN-2 on 30 Nov., 2012. During the time interval between 16:11:30 and 16:12:30 UT, three to four clear stripes were observed between 16.0 and 19.0 MHz. The total duration of the event was approximately $1 \mathrm{~min}$.

Two groups of ZPs were observed on 1 Jan., 2014 (Fig. 1d). The first group with up to five stripes was detected between 21:59:35 and 22:00:35 UT in the frequency range 15-17.5 MHz. The second group was detected at higher frequencies, 19.521.5 MHz, during the time interval 22:01:30-22:02:15 UT. 

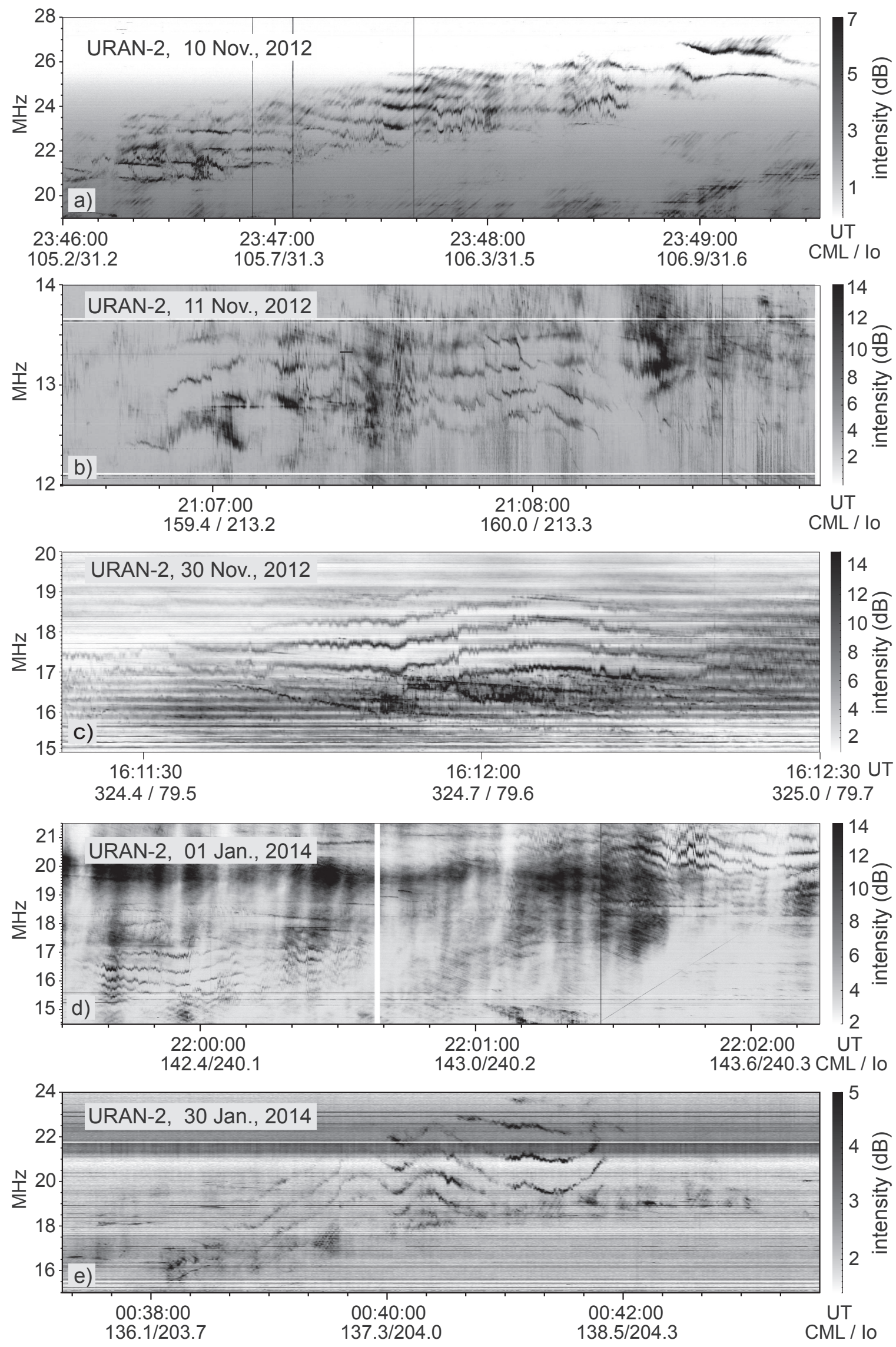

Fig. 1. Examples of ZPs observed by URAN-2 radio telescope. The dynamic spectra show the total intensities $I$ (see Eq. (1)) of radio emission in $\mathrm{dB}$ above the frequency-dependent background noise of the receiver. This background is defined as a time-averaged frequency profile of a broadband noise injected from a reference noise oscillator into the receiving path before and at the end of each observing session. 

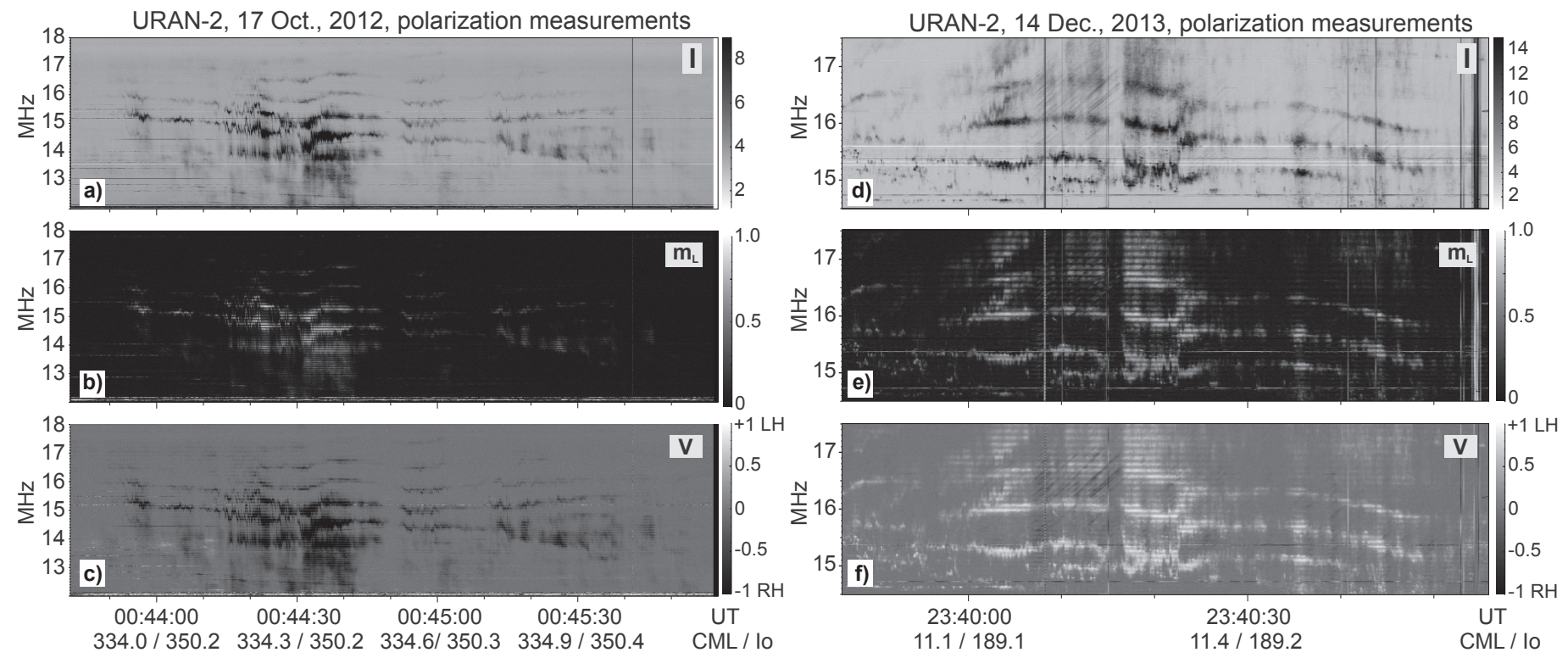

Fig. 2. URAN-2 polarization measurements of ZP in Jovian DAM. Dynamic radio spectra in panels $a$ and $d$ show the intensity of the radio emission in $\mathrm{dB}$. Panels $b$ an $e$ : degree of linear polarization $\left(m_{\mathrm{L}}=\sqrt{\left(Q^{2}+U^{2}\right)} / I\right)$ and panels $c$ and $f$ : normalized degree of circular polarization $(Q, U$, and $V$ are the Stokes parameter). The dark color in panels $c$ and $f$ defines the right hand polarization $(\mathrm{RH})$ and bright color indicates the left hand polarization $(\mathrm{LH})$. Horizontal fringes alternately bright and dark in spectra are due to the Faraday rotation.

Table 1. URAN-2 Observation campaigns of Jupiter's radio emission.

\begin{tabular}{ccccc}
\hline \hline Camp. No. & Begin & End & Sessions & ZP \\
\hline 1 & 18 Sep. 2012 & 23 May 2013 & 214 & 18 \\
2 & 21 Aug. 2013 & 31 Mar. 2014 & 212 & 23 \\
3 & 25 Nov. 2014 & 31 May 2015 & 153 & 10 \\
\hline
\end{tabular}

Notes. The begin and end of each campaign as well as number of observation sessions are indicated. The last column shows the number of observed ZP events.

Finally, Fig. 1e shows the example of weaker ZPs observed on 30 Jan., 2014, 00:38:40-00:42:00 UT in the frequency range 16-23 MHz. Four stripes were detected simultaneously. The intensity of the ZPs was about $10 \mathrm{~dB}$ lower than in Figs. $1 \mathrm{~b}$ and $\mathrm{d}$.

Flux densities of the zebra stripes normalized to a distance of 1 AU were estimated to be in the range between $\sim 10^{5}$ and $10^{6} \mathrm{Jy}$ (Jansky) at 12-27 MHz. For comparison, this range of flux density is 1-2 orders of magnitude less than the strongest component of the Jovian radio emissions, the rotational-averaged flux densities of the Io controlled DAM (Zarka 1998; Zarka et al. 2004).

The spectral structure shown in Fig. 1 is similar to the socalled ZPs observed in the dynamic spectra of sporadic solar radio emission (at frequencies from hundreds of $\mathrm{MHz}$ to a few $\mathrm{GHz}$, Chernov 2010) and to the quasi-parallel stripes observed at low frequencies of Jovian kilometric radio emission (tens of $\mathrm{kHz}$ ) by Cassini spacecraft during its flyby of Jupiter (Kurth et al. 2001). The other examples of observed ZPs are shown in Figs. 2, 5, and 6 as well as in Litvinenko et al. (2016, Fig. 8).

\subsection{Polarization}

Operated in the polarization mode, the digital receiver of URAN-2 (DSPz) receives the signals from two orthogonal polarizations separately. These two input signals (or input voltages) $V_{A}$ and $V_{B}$ are processed by the digital spectro-polarimeter producing four parallel output spectra: two autocorrelation spectra or total intensities $I_{A}=\left\langle V_{A} V_{A}^{*}\right\rangle$ and $I_{B}=\left\langle V_{B} V_{B}^{*}\right\rangle$ as well as real $C_{A B}^{r}=\mathfrak{R}\left(\left\langle V_{A} V_{B}^{*}\right\rangle\right)$ and imaginary $C_{A B}^{i} \mathfrak{I}=\left(\left\langle V_{A} V_{B}^{*}\right\rangle\right)$ parts of cross-correlation spectra between the input signals, where asterisks denote complex conjugate and $\langle\ldots\rangle$ is a time-averaging operation. Then the Stokes parameters can be calculated as:

$$
\begin{aligned}
I & =I_{A}+I_{B} \\
Q & =I_{A}-I_{B} \\
U & =2 C_{A B}^{r}-I_{A}-I_{B} \\
V & =2 C_{A B}^{i}-I_{A}-I_{B},
\end{aligned}
$$

where $I$ is the total intensity of the radio emission, $Q$ and $U$ are the linear polarization parameters and $V$ is the circular polarization component. The Stokes parameters determine the axial ratio as well as orientation of the polarization ellipse (Krauss 1966, pp. 109-129).

The polarization properties of the ZPs were investigated using the polarization measurement capability of the URAN-2 telescope. Figure 2 shows the dynamic spectra of the normalized Stokes parameters (intensity $I$, degree of the linear polarization $m_{\mathrm{L}}=\sqrt{\left(Q^{2}+U^{2}\right)} / I$, and circular polarization $\left.V / I\right)$ of the ZPs observed on 17 Oct., 2012 and 14 Dec., 2013. The spectra show that the events are highly polarized radio emission. According to the IRE definition 1942 (Krauss 1966) negative values of circular polarization parameter $V<0$ denote the right-handed (RH) and positive ones $V>0$ correspond to the left-handed (LH) polarized radio emission. Therefore, the ZP recorded on 17 Oct., 2012 is right-hand polarized and ZP observed on 14 Dec., 2013 is left-hand polarized radio emission.

Although the URAN-2 is able to measure the full polarization state of the radio emission (all Stokes parameters), we did not determine the exact degree of total, circular, and linear polarization of ZPs in the present study. The reason is that the calibration of the polarization response of URAN-2 is currently in progress and not yet finished, and therefore, we cannot provide the reliable polarization parameters. In our study we used the parameter of circular polarization $V$ only for determination of the polarization sense, that is, right-hand or left-hand. Nevertheless, 
Table 2. Outlier events of the ZPs.

\begin{tabular}{ccccc}
\hline \hline Date & Pol. & CML & Io ph. & Source \\
\hline 26 Oct. 2012 21:19 UT & RH & 278 & 197 & Io-A \\
17 Oct. 2012 00:43 UT & RH & 334 & 350 & non-Io-A \\
15 Oct. 2012 23:24 UT & LH & 135 & 136 & Io-D \\
15 Oct. 2012 23:36 UT & LH & 142 & 138 & Io-D \\
23 Dec. 2013 21:00 UT & LH & 191 & 199 & non-Io-D \\
23 Dec. 2013 21:03 UT & LH & 193 & 200 & non-Io-D \\
23 Dec. 2013 21:14 UT & LH & 199 & 201 & non-Io-D \\
\hline
\end{tabular}

Notes. Date, polarization, CML, and Io phase coordinates are indicated. The last column shows the possible source of the ZP.

the clear Faraday fringes due to Faraday rotation (change in orientation of the polarization ellipse of radio wave measured by linear polarized antennas) are easily visible as bright and dark horizontal stripes in the dynamic spectra of the Stokes parameters in Fig. 2. The ZPs are modulated by these fringes. These Faraday fringes occur on the dynamic spectra when the highly elliptically polarized radio emission is observed by linearly polarized antennas. Therefore, we can conclude that the ZPs similar to Jovian DAM (Dulk et al. 1992; Shaposhnikov et al. 1997) are elliptically polarized. It is also worth noting that the fringes are not visible in the dynamic spectra of intensities $(I)$ in Figs. 1 and 2 because the presented total intensity $I$ is a sum of intensities measured by two linearly polarized orthogonal antenna dipoles of URAN-2, that is, $I=I_{A}+I_{B}$. Since the Faraday fringes observed in $I_{A}$ are in anti-phase (with respect to the frequency) to fringes in $I_{B}$ the dynamic spectrum $I=I_{A}+I_{B}$ does not exhibit any Faraday rotation. In fact, the Faraday fringes should not be visible in the spectra of the Stokes parameter $V$ (panels c and $\mathrm{f}$ ) because the Faraday rotation modifies only the direction of the linear polarization but not the rate of the circular polarization. Nevertheless, the fringes are also observed in the circular polarization plots due to an imperfect calibration of the URAN-2 polarization response, as mentioned above.

Assuming, that the ZPs propagate as a right-hand extraordinary R-X mode (similar to the DAM), the sign of circular polarization $V$ can be used for subsequent separation between the emission radiated from the Northern or Southern magnetic hemisphere of Jupiter. The R-X waves from the Northern sources are observed as right-hand while the emissions from the Southern sources are observed as left-hand polarized radio emission.

The polarization measurements of the ZPs reveal relatively even distribution of left-hand and right-hand polarized events, that is, 29 events as RH emission and 22 events as LH radiation. The polarization of the events is not related with certain periods of time. The measurements show that the ZPs can be associated with both magnetic hemispheres of Jupiter. Additionally, the RH polarized events consist of larger numbers of stripes within a $\mathrm{ZP}$, that is, from 3 to 9 individual stripes in each event, while $\mathrm{LH}$ events are comprised of 3 to 7 individual stripes.

\subsection{Distribution of the observed ZP in CML (System III 1965) and lo phase coordinates}

Generally, the occurrence probability of the Jovian radio emission depends on the Jovian CML (System III 1965) and Io-phase coordinates of the observer. We have defined the CML and Io phase at the central time of each particular zebra even. The corresponding distribution of the occurrence of the right- $(\mathrm{RH})$ and left-handed (LH) polarized zebra events in CML - Io phase co-

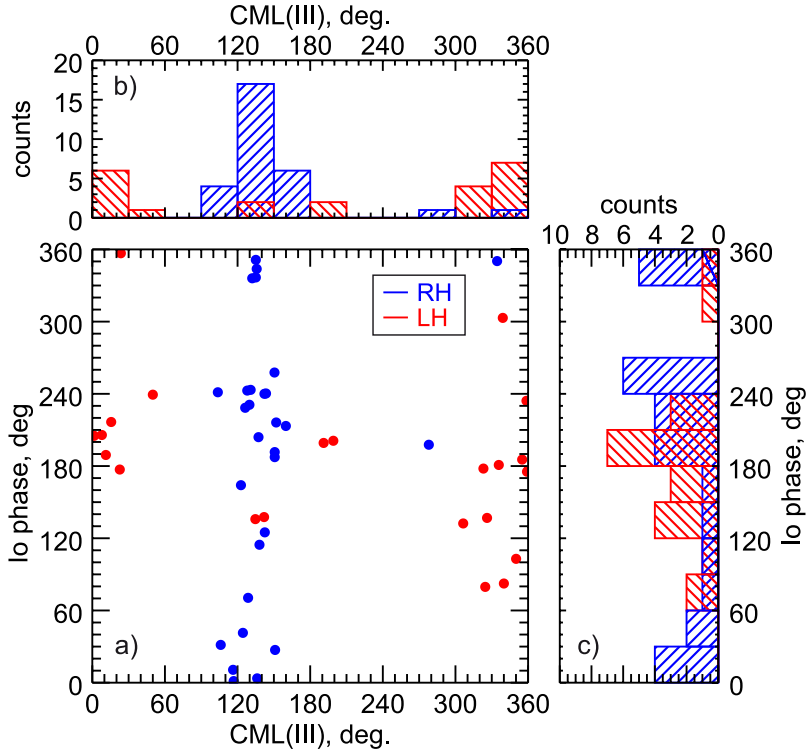

Fig. 3. Statistical results of $51 \mathrm{ZPs}$ observed by URAN-2 telescope. Panel $a$ : occurrence of the ZPs in CML - Io phase coordinates. Blue color indicates the right-handed polarized events (northern sources) and the left-handed polarized events (southern sources) are marked by red color. The top panels show the histogram of a CML's (panel $b$ ) and the right panel $c$ is a histogram of Io phases for $\mathrm{RH}$ (blue) and $\mathrm{RH}$ (red) events. The bin size is $30^{\circ}$.

ordinates is shown in Fig. 3a. The top panel (Fig. 3b) shows histograms of CMLs and the right panel (Fig. 3c) is the histograms of Io phases of the RH and LH zebra events. We note the strong correlation between the CML position of the observer and the occurrence of the ZPs. In particular, the histograms in Fig. $3 b$ clearly show the two separate source regions on the Northern (RH events) and the Southern (LH events) hemisphere. The northern ZPs, except several outlier events which are discussed below, were observed in the sector of CMLs from $90^{\circ}$ to $180^{\circ}$ with a clear maximum in the range $120^{\circ}-150^{\circ}$. At the same time, most of the southern events were recorded between $300^{\circ}$ and $60^{\circ}$ (via $360^{\circ}$ ) of CML with a maximum around $330^{\circ}-360^{\circ}$. Maxima of the RH and LH distributions are shifted by $\sim 200^{\circ}$. The histograms in Fig. 3c show no evident correlation between $\mathrm{ZP}$ occurrence and the position of the Io moon. Thus we can conclude that zebra events are non-Io controlled radio emission.

As shown in Fig. 3, most of the events are observed in two separate CML regions, except for several ZP events, which are listed in Table 2. In particular, two RH ZPs were observed outside of the main distribution at $278^{\circ}$ and $334^{\circ}$ of CML and five LH ZPs were detected at $135^{\circ}, 142^{\circ}, 191^{\circ}, 193^{\circ}$, and $199^{\circ}$ of CML. Here it is worth noting that most of the ZPs were observed as single events with clear onset and end. Nevertheless, sometimes the zebra events were observed in groups, that is, single events were detected consecutively during a short time interval (a couple of minutes or tens of minutes; see example in Fig. 1d). Therefore, such events have practically the same CML and Io phase coordinates in Fig. 3. In total, six groups, (15 single events in total) were recorded. As listed in Table 2, five of the $\mathrm{LH}$ outlier events with CML coordinates around $130^{\circ}-140^{\circ}$ and around $190^{\circ}$ were observed in two groups on 15 Oct., 2012 (two events), and 23 Dec., 2013 (three events). We found that an "outlier" RH event on 26 Oct., 2012, was observed together with strong Io-A burst of DAM (Io-A source of DAM). The emission from Io-A source is $\mathrm{RH}$ polarized and corresponds to sources in the Northern hemisphere when CML ranges around $200^{\circ}-270^{\circ}$ 

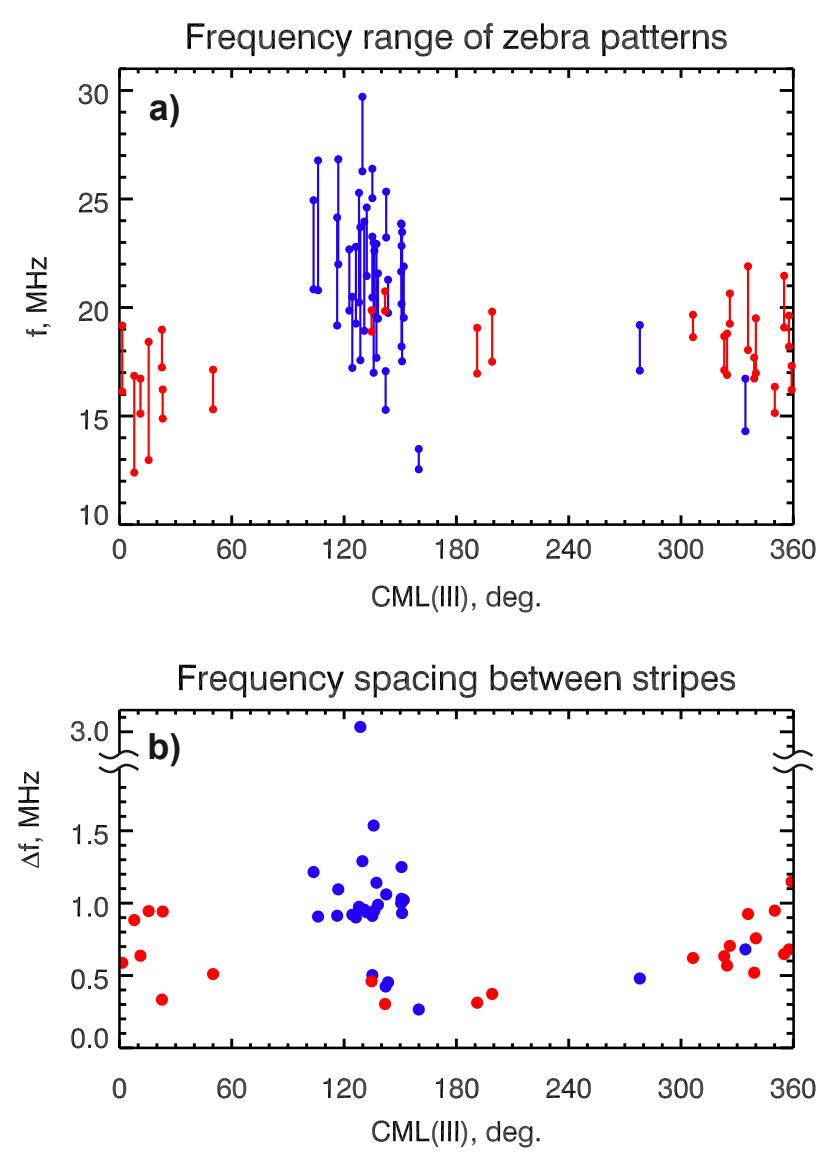

Fig. 4. Frequency range (panel $a$ ) and frequency intervals $\Delta f$ between stripes (panel $b$ ) as a function of CML. The red color indicates LH polarized ZPs and blue denotes RH polarized events. The lower markers on the bars, which denote one event in panel $a$, represent the minimum frequency and the upper markers indicate the highest recorded frequency of the zebra event. We note that there is the $y$-axis breaking between 1.8 and $2.9 \mathrm{MHz}$ in panel $b$.

and Io has a phase around $205^{\circ}-260^{\circ}$, (e.g., Carr et al. 1983; Marques et al. 2017). The other two outlier LH events, 15 Oct., 2012, 23:24 UT and 15 Oct., 2012, 23:36 UT were observed in the latter part of strong Io-D arc of DAM, which is LH polarized emission from Southern hemisphere observed in CML between around $90^{\circ}-200^{\circ}$ and Io phase around $95^{\circ}-130^{\circ}$. Thus we can suggest that these three outlier events may be part of Iocontrolled DAM while the histograms in Fig. 3 show that most of the observed ZPs are non-Io DAM. Nevertheless additional observations will be needed to verify whether or not ZPs can be associated also with Io-controlled DAM. The other four outlier events (17 Oct., 2012 and 23 Dec., 2013) were not observed with Io-controlled DAM.

\subsection{Frequency range, bandwidth, and frequency splitting between stripes}

ZPs were observed in overall frequency range from 12.5 to 29.7 MHz. Figure 4a shows the frequency ranges of all $51 \mathrm{ze}-$ bra events as a function of CML. One bar denotes one event. The lower marker on the bar represents the minimum frequency in the lowest stripe and the upper marker indicates the highest recorded frequency in the highest stripe of the event. Moreover, the events are marked by color in accordance to the sense of polarization (blue: RH, red: LH polarized). As seen in Fig. 4a, the
RH ZPs are spread over a wider frequency range compared to LH events. The northern ZPs were observed in the frequency range from 12.5 up to $29.7 \mathrm{MHz}$ while the southern ones were recorded in frequency range from $15.1 \mathrm{MHz}$ to $21.9 \mathrm{MHz}$. This is also consistent with distribution of the surface (1-bar pressure level) magnetic field of Jupiter and therefore, with the maximum electron cyclotron frequency. As was shown by Genova \& Aubier (1985) and Hess et al. (2011) the maximum radio frequency for the northern Io-DAM (Io-A and Io-B sources) is $\approx 38 \mathrm{MHz}$ while the southern Io-DAM sources (Io-C and Io-D) are mainly observed at lower frequencies $<25 \mathrm{MHz}$.

Another important parameter is the frequency interval $\Delta f$ between neighboring stripes, which may shed light on the generation mechanism of ZPs. For instance, the mechanism of excitation of harmonically related plasma waves, such as Bernstein modes, predicts the frequency equidistant stripes. At the same time, the non-equidistant stripes can be explained by inhomogeneous models such as double plasma resonance (DPR), in which the sources of each zebra stripe are spread out along magnetic field line. Additionally, according to the DPR model, the distances between stripes depend on the gradients of plasma density and magnetic field in the source.

For each event we defined the frequency splitting $\Delta f$ as a frequency interval between intensity maxima of the neighboring stripes in the frequency profile. Figure $4 \mathrm{~b}$ shows the averaged (for each ZP event) $\Delta f$ between the stripes vs. CML (System III (1965)) of the observer. The defined $\Delta f$ for 50 out of all 51 events ranges from 0.26 to $1.5 \mathrm{MHz}$. Only in one case (1 Feb., 2014, see Fig. 5) was the averaged frequency splitting significantly higher $\Delta f=3 \mathrm{MHz}$. Figure $4 \mathrm{~b}$ also shows that $\Delta f$ for RH polarized (northern sources) ZPs was generally larger than for LH (southern sources) zebras. In 23 out of $29 \mathrm{RH}$ events, the frequency splitting was $\Delta f>0.9 \mathrm{MHz}$, while for all $\mathrm{LH}$ events, $\Delta f$ ranges from 0.26 to $0.9 \mathrm{MHz}$. The mean value of frequency splitting for RH events (except of 1 Feb., 2014) is $\overline{\Delta f}=0.9 \pm 0.3 \mathrm{MHz}$ and for LH ZPs $\overline{\Delta f}=0.6 \pm 0.2 \mathrm{MHz}$. The frequency splitting between the stripes in one single event varies in time while Fig. $4 \mathrm{~b}$ shows only the averaged values for each of the events.

In fact, the stripes in ZPs are not exactly equidistant. Analyses have shown that in most cases the frequency splitting slightly increases with the emission frequency. This was observed in 43 events. Nevertheless, in 6 events the frequency splitting between stripes decreases with increasing emission frequency. This is unusual for the ZPs observed in a solar radio emission, where the frequency intervals between stripes always increases with the frequency of emission. It is interesting that all six events with decreasing frequency splitting were observed as LH emission, that is, from the Southern hemisphere. Moreover we have also found two events in which the frequency intervals decrease with the emission frequency in an earlier part of the considered events and then $\Delta f$ becomes wider in the latter half of ZP spectra.

The instantaneous bandwidth $\mathrm{d} f$ of one stripe defined as full width at half maximum of the frequency profile varies from 0.1 to $0.2 \mathrm{MHz}$, that is less than the frequency spacing $\Delta f$ between the stripes.

In all the examples, the stripes exhibit rapid wave-like frequency drift, remaining quasi-equidistant to one another. These temporal frequency oscillations do not occur simultaneously for all the stripes, as shown in Fig. 5. On the zoomed part of the ZP spectra, observed on 14 Mar., 2015, one can see the small time shift of about $0.1 \mathrm{~s}$ between the intensity profiles of each consecutive stripe towards the lower frequencies. The other dynamic radio spectra exhibit a similar delay, of $0.1-0.2 \mathrm{~s}$ or $1-2$ pixels, 


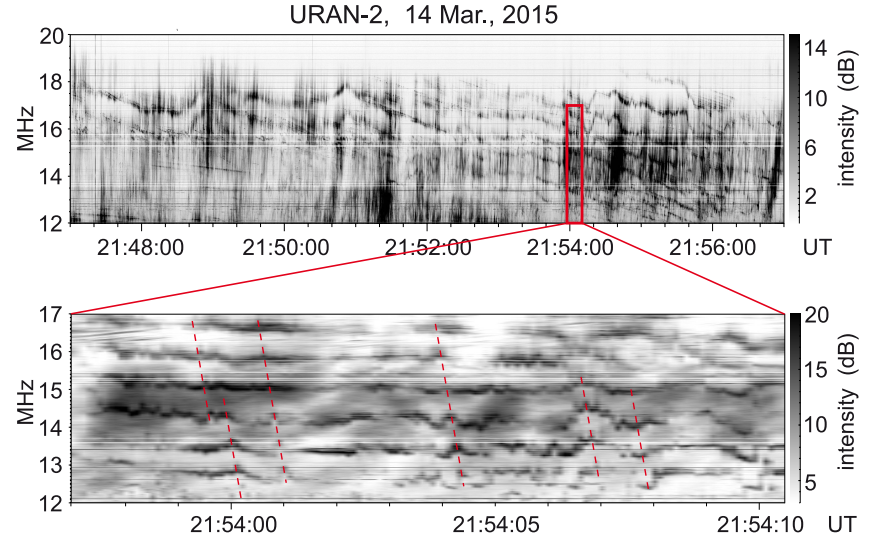

Fig. 5. Example of the rapid oscillation in frequency of the stripes. Top panel: ZPs observed on 14 Mar., 2015, at 21:47:00-21:57:00 UT in the frequency range 12-20 MHz. Bottom panels: zoomed part (21:53:5721:54:10.5 UT and 12-17 MHz) of the radio spectrum. Each consecutive zebra stripe is shifted by $1-2$ pixels, or $100-200 \mathrm{~ms}$, relative to the next stripe at lower frequency.

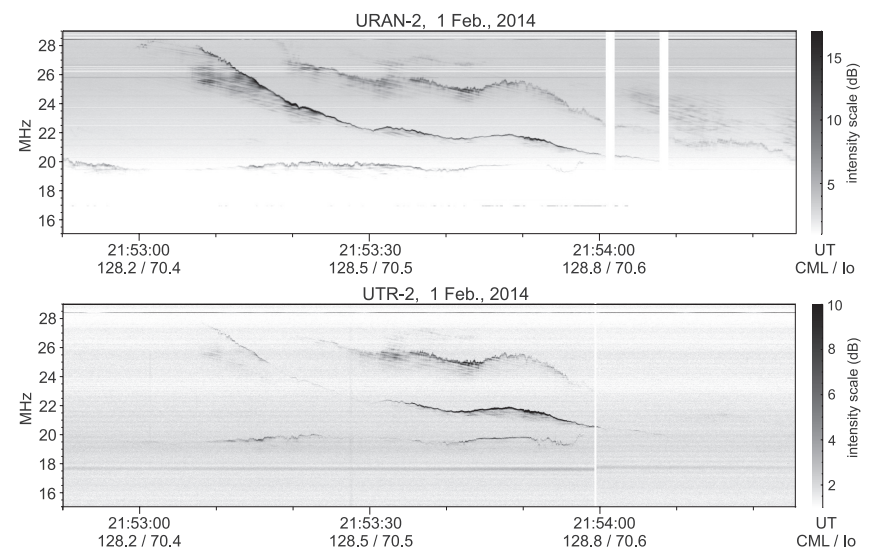

Fig. 6. Simultaneous coordinated observations of Jupiter by URAN-2 (top panel) and UTR-2 (bottom panel) radio telescopes. Both telescopes recorded ZP event between 21:53 and 21:54 UT.

in the radio spectra (more accurate measurements of this delay require better time resolution of the radio spectra, which was $0.1 \mathrm{~s}$ ). This strongly suggests that each stripe of the ZPs originates from a different location along the magnetic field line and the frequency oscillations may be due to the substantial motions of the density gradient along the magnetic line.

\subsection{Simultaneous coordinated observations of ZP with UTR-2 and URAN-2 radio telescopes}

One of the problems of low-frequency radio astronomy is the distortion of the signal by ionospheric scintillations. Plasma irregularities in the Earth ionosphere cause diffraction and scattering of the radio waves, and therefore, the recorded signal presents random temporal amplitude and phase variation. Ionospheric scintillations increase with decreasing signal frequency and then reach a maximum close to the ionospheric cut-off frequency $(8-10 \mathrm{MHz})$. In the decametric frequency range, ionospheric scintillations manifest themselves as intense interference fringes in the dynamic spectrum. Sometimes these fringes are observed as frequency quasi-equidistant stripes, similar to the reported ZPs. In most cases it is easy to distinguish between ionospheric scintillation and ZPs, while, in contrast to the ZPs, the instantaneous bandwidth of the interference fringes is larger than frequency distance between the consecutive fringes (see Figs. 10 and 11 in Konovalenko et al. 2016).

One of the very effective ways to avoid ionospheric scintillations is simultaneous observations of the radio source by several telescopes separated in space. In this case, each of the telescopes will observe the radio emission through different ionospheric plasma and therefore, the ionospheric interference will be different. Then comparing the simultaneously observed radio events it is easy to decide which parts of the dynamic spectra are the result of the ionospheric scintillations and which ones are the original signal.

During the second observation campaign from Aug., 2013, to Mar., 2014, we organized one month of simultaneous observation by URAN-2 and UTR-2 radio telescopes, located about $160 \mathrm{~km}$ from each other. Fortunately, during these simultaneous observations, one event of ZP was recorded. As shown in Fig. 5, URAN-2 observed three stripes in frequency range from 20 to $28 \mathrm{MHz}$, and UTR-2 observed similar spectral features. These observations allow us to exclude the eventual possibility that ZPs are produced by the terrestrial ionosphere.

\section{Discussion}

The fine structure known as zebra structure was first observed against the continuum background of solar type IV radio bursts in the meter and decimeter wavelengths as a system of quasiparallel drifting stripes of enhanced brightness. Recently, ZPs have also been observed in the solar radio emission at centimeter wavelengths (Altyntsev et al. 2007). These structures, similar the zebra structures, are observed in the very low frequency (VLF) hisses in the Earth's magnetosphere (Titova et al. 2007) and in the microwave radiation of a pulsar in the Crab nebula (Hankins \& Eilek 2007). There are many papers devoted to the origin of the zebra structure in the dynamic spectra of the solar radiation. The main part of theories devoted to the origin of the zebra structure use the plasma mechanisms of emission generation where the plasma (electrostatic) waves or whistlers are generated with following transformation of these waves into electromagnetic radiation (see, e.g., Zheleznyakov \& Zlotnik 1975; Chernov 1976). Some theories consider interference as a reason for zebra structure origin in the dynamic spectra, which appears in the case of wave propagation in plasma with regular density inhomogeneities (Barta \& Karlicky 2006; Ledenev et al. 2006). Reviews of these papers can be found in Chernov (2006, 2010); Zlotnik (2009).

The most acceptable theory for the generation of the $\mathrm{ZP}$ in the solar radio emission is based on the DPR effect at the electron cyclotron harmonics (Zheleznyakov \& Zlotnik 1975; Kuijpers 1975; Zheleznyakov et al. 2016). According to this theory, in an inhomogeneous magnetic trap filling the weakly anisotropic plasma, which satisfies the condition $f_{\mathrm{pe}} \gg f_{\text {ce }},\left(f_{\mathrm{pe}}\right.$ and $f_{\text {ce }}$ are plasma and cyclotron frequencies of electrons, accordingly) the plasma waves are excited at the frequency of upper hybrid resonance, $f_{\mathrm{UH}}$, due to the electrons with unstable velocity distribution (e.g., the loss-cone distribution). In the regions where the frequency of upper hybrid resonance coincides with the electron cyclotron harmonics $f_{\mathrm{UH}}=\sqrt{f_{\mathrm{pe}}^{2}+f_{\mathrm{ce}}^{2}} \simeq s f_{\mathrm{ce}}$ ( $s$ is the harmonic number) the level of excited plasma waves strongly increases.

The ZPs observed in the solar and Jupiter's radio emission demonstrate some similar spectral properties. A comparative morphological analysis for the parameters of the zebra structures in the solar and the Jovian decametric radio emission was 
first presented in a paper by Litvinenko et al. (2016). The analogy between the ZPs in the solar and the Jovian radiation leads one to suppose that DPR mechanism may be responsible for the generation of the ZPs in the Jovian decametric radio emission. Kuznetsov \& Vlasov (2013) considered the DPR as a possible mechanism of formation of the striped structures observed by Cassini spacecraft in the Jovian kilometer radiation (tens of $\mathrm{kHz}$ ). They modeled the qualitative emission spectrum with zebra structures and proposed the location of the sources along field lines in Io plasma torus. Their model explains the striped spectrum, but contradicts the observations: the strip frequency spacing decreases with emission frequency while the observed spacing increases with frequency.

If we assume that the ZP emission in Jovian DAM is generated by the DPR at the electron cyclotron harmonics then it is easy to estimate the required plasma densities to satisfy the DPR condition. As was mentioned in Sect. 3.3, the ZPs were observed in the frequency range between 12.6 and $29.7 \mathrm{MHz}$. In order to satisfy the DPR resonance condition, the electron number densities along the magnetic field line have to be $1.9 \times$ $10^{6} \mathrm{~cm}^{-3}-1.1 \times 10^{7} \mathrm{~cm}^{-3}$. At the same time, Voyager and Galileo radio occultation measurements showed that the main electron peak density in the Jovian ionosphere is about $2-2.3 \times 10^{5} \mathrm{~cm}^{-3}$ at altitudes of $0.027 R_{\mathrm{J}}$ (Hinson et al. 1997, 1998). Therefore, the estimated electron number densities required for the generation of zebra structures are several orders of magnitude larger than the peak of the electron number density in the Jovian magnetosphere. The required densities are also significantly higher than peak plasma densities in the Io plasma torus, that is, $2 \times 10^{3} \mathrm{~cm}^{-3}$ based on Voyager 1 observations (see, e.g., Bagenal 1994) as well as in regions near Io ionosphere where the peak electron densities ranged from 2.1 to $6.8 \times 10^{4} \mathrm{~cm}^{-3}$ (Gurnett et al. 2001). Moreover, unlike the solar corona, Jupiter's plasma is strongly anisotropic, that is, $f_{\mathrm{pe}} \ll f_{\mathrm{ce}}$, in most of the regions of the magnetosphere. Therefore, the mechanism of DPR with electrons cannot explain our observations since it requires extremely high plasma density which is very unlikely in Jovian magnetosphere.

Some proposed mechanisms of $\mathrm{ZP}$ generation in the solar radio spectra involve the presence of energetic ions or ion structures in the source region. Treumann et al. (2011) discussed a model of the zebra in solar flare radio emission in which continuum radio bursts may be modulated by the harmonics of the ion cyclotron frequency $f_{\mathrm{ci}}$ under certain restrictive conditions (presence of the strong-field-aligned electric fields and weakly relativistic ions). In this case, the distance between the stripes corresponds to the harmonics of $f_{\mathrm{ci}}$. The typical values of $f_{\mathrm{ci}}$ in the inner Jovian magnetosphere are tens of $\mathrm{kHz}$ while the observed distances between zebra structures in DAM are $0.3-1.5 \mathrm{MHz}$ (Fig. 4b). Therefore the theory proposed by Treumann et al. (2011) cannot explain the observed ZPs in the DAM.

Recently, Zlotnik et al. (2016) proposed an alternative mechanism of zebra structure formation in Jovian kilometer radiation which was observed by Cassini at low frequencies $(30-70 \mathrm{kHz}$, Kurth et al. 2001). The model is based on the DPR at the ion cyclotron harmonics. The mechanism involves the excitation of the ion cyclotron waves at low hybrid frequency and then a non-linear transformation of this low-frequency emission to the high-frequency wave due to coalescence process with a high frequency mode, for example, plasma upper hybrid frequency of the electrons. In the mechanism of DPR with ions, the low hybrid plasma waves are enhanced in regions where the low-hybrid resonance frequency $f_{\mathrm{LH}}$ coincides with the harmonics of $f_{\mathrm{ci}}$. In contrast to the DPR with the electrons, the DPR with the ions can be operated in the regions where $f_{\mathrm{pe}} \ll f_{\text {ce }}$, which is fulfilled in most of the regions of the Jovian magnetosphere. Therefore the DPR effect at the ion cyclotron harmonics can be realized for much less plasma density than the DPR effect at the electron cyclotron harmonics. In this case, $f_{\mathrm{LH}} \approx f_{\mathrm{pi}}\left(f_{\mathrm{pi}}\right.$ is the ion plasma frequency) and the resonance condition is $f_{\mathrm{LH}}=s f_{\mathrm{ci}}$. Due to the existence of the plasma density gradient (non-uniform radio source) the resonance condition for each harmonic $s$ is satisfied at different altitude. Produced by the DPR effect at the ion cyclotron harmonics, the low-frequency plasma waves cannot escape the source and should be first transformed to the highfrequency electromagnetic waves $\left(f_{\mathrm{em}}\right)$ in a non-linear coalescence process with a high-frequency mode (e.g., with plasma waves at upper hybrid frequency). Coalescence of these waves $\left(f_{\mathrm{em}}=s f_{\mathrm{ci}}+f_{\mathrm{ce}}\right)$ results in generation of electromagnetic waves with a spectrum in the form of ZP (see Zlotnik et al. (2016) for more details).

Since in the DPR with ions, the plasma waves are excited at low frequencies of the $f_{\mathrm{ci}}$ harmonics, the required densities of the ions are significantly lower than for DPR with the electrons. Using the model of the Jovian magnetic field, for example, VIP4 model, we can calculate the local $f_{\text {ce }}$ along the magnetic field line. For example, for the field line with latitude $49.6^{\circ}$ and longitude $170^{\circ}$ (in System III 1965) $f_{\text {ce }}=12.5 \mathrm{MHz}$ will be at $\sim 0.34 R_{\mathrm{J}}$ above Jupiter's surface and for $f_{\text {ce }}=29.7 \mathrm{MHz}$ at the altitude $\sim 0.054 R_{\mathrm{J}}$. At these altitudes, the ion cyclotron frequencies are $f_{\mathrm{ci}}=6.8 \mathrm{kHz}\left(\right.$ at $0.34 R_{\mathrm{J}}$ ) and $f_{\mathrm{ci}}=16.2 \mathrm{kHz}$ (at $0.054 R_{\mathrm{J}}$ ). Thus using the resonant condition $f_{\mathrm{pi}}=s f_{\mathrm{ci}}$ the ion number densities for the second harmonic ( $s=2$, the minimal possible harmonic in the DPR) have to be $4.2 \times 10^{3} \mathrm{~cm}^{-3}$ and $2.4 \times 10^{4} \mathrm{~cm}^{-3}$ correspondingly for the altitudes $0.34 R_{\mathrm{J}}$ and $0.054 R_{\mathrm{J}}$. These ion densities are more realistic. For example, Voyager 2 occultation measurements (Fig. 4 in Hinson et al. 1998) show that the number density of the electrons at altitude $0.054 R_{\mathrm{J}}$ is $\approx 1.2 \times 10^{4} \mathrm{~cm}^{-3}$, that is, comparable to the densities estimated above ion densities (assuming that primary ions in the upper Jovian atmosphere are $H^{+}$and that the number density profile of $\mathrm{H}^{+}$ions should be similar to the electron density profile due to quasi-neutrality of the magnetospheric plasma).

Therefore the DPR mechanism with ions can potentially exist in the upper Jovian ionosphere. Nevertheless additional theoretical investigations are needed to explain the modes conversion from low-frequency ion cyclotron waves to the highfrequency electromagnetic waves. In particular, the non-linear coalescence process should explain how to reach the observed intensities of the ZPs (just 1-2 orders lower than Jovian DAM, which is the strongest Jovian radio emission).

\section{Conclusions}

We report the first systematic study of the stripe-like zebra patterns (ZPs) in decametric frequency range of Jovian radio emission. The ZPs, the detection of which we first reported in Litvinenko et al. (2016), are observed as a number of quasiharmonically related stripes of the enhanced brightness with fast parallel drift in time. The performed analysis of the observations has allowed us to conclude that the observed ZPs are a new type of narrow band spectral structures in Jovian DAM. The main results of the observations are summarized below:

1. In total $51 \mathrm{ZP}$ events were detected during three observational campaigns (Sep., 2012-May 2013, Aug., 2013-Mar., 2014 and Nov., 2014-May, 2015) on URAN-2 (Poltava, Ukraine) radio telescope. 
2. ZPs are observed as a number of quasi-equidistant stripes (from 3 to 9) of the enhanced brightness drifting in parallel in time. The number of stripes varies with time.

3. ZPs events are detected in decametric frequency range from 12.5 to $29.7 \mathrm{MHz}$. The emission frequency intervals of stripes vary with time.

4. The minimum duration of one single ZP event was $20 \mathrm{~s}$ and the maximum was $4 \mathrm{~min} 50 \mathrm{~s}$. The mean duration averaged over all zebra events was $120 \mathrm{~s}$.

5. The flux densities of the zebra stripes normalized to a distance of $1 \mathrm{AU}$ are $\sim 10^{5}-10^{6} \mathrm{Jy}$ (Jansky) at $12-27 \mathrm{MHz}$ that is 1-2 orders lower than intensities of the Io controlled DAM. The flux densities of ZP stripes vary significantly in time and frequency.

6. Strongly polarized radio emission.Twenty-nine events were observed as RH polarized emission and 22 events as LH radiation. Assuming that the ZPs propagate as R-X wave mode, we conclude that the sources can be located in both Northern (RH events) and Southern (LH events) magnetic hemispheres of Jupiter. The RH events have a larger number of stripes (from 3 to 9) than the LH events (from 3 to 7).

7. ZPs are detected in two active sectors of Jovian CML's: $100^{\circ}$ to $160^{\circ}$ for Northern sources and $300^{\circ}$ and $60^{\circ}$ (via $360^{\circ}$ ) for the Southern ones. ZPs are non Io-DAM while no dependency with Io position was detected.

8. The frequency interval $\Delta f$ between neighboring stripes (or frequency splitting) in the most cases was from 0.26 to 1.5 MHz. $\Delta f$ for the Northern sources (RH events) is generally larger than for the Southern ones (LH events). The frequency splitting between the stripes in one single event varies in time. $\Delta f$ can increase (in 43 out of 51 events) as well as decrease (in 6 events) with frequency. In two events, $\Delta f$ was observed to decrease with the emission frequency at the beginning of the event and then to increasing in the latter part of the event.

9. The instantaneous bandwidth $\mathrm{d} f$ of the stripes varies from 0.1 to $0.2 \mathrm{MHz}$, that is, less than the frequency splitting $\Delta f$ between the stripes.

10. Zebra stripes exhibit rapid temporal wave-like frequency drifts. There is a small time delay (100-200 ms) between the profiles of each consecutive stripe towards lower frequencies that supports the suggestion that the source of each stripe is located at different locations, most probably along the same magnetic field line.

11. Simultaneous observations by URAN-2 and UTR-2 evidently show that ZPs are not a product of the Earth's ionospheric scintillations.

12. We discussed the double plasma resonance with electrons or ions as a possible source of the ZPs. The DPR with electrons requires significantly higher plasma densities than those observed in the Jovian magnetosphere. The DPR with ions can potentiality exist in the upper Jovian ionosphere. Nevertheless, additional theoretical studies are needed to clarify the non-linear modes conversion from the low-frequency ion cyclotron waves to the high-frequency electromagnetic waves.

Acknowledgements. This work was financed by the Austrian Science Fund (project P23762-N16). Work of S.R. and J.S. was supported by the Oelzelt foundation of the Austrian Academy of Sciences. V.E.S. acknowledges the financial support of the Russian Foundation for Basic Research (project 16-02-00373-a, 17-02-00091). Data are available upon request from corresponding author.

\section{References}

Abranin, E. P., Bruck, Y. M., Zakharenko, V. V., \& Konovalenko, A. A. 2001, Exp. Astron., 11, 85

Altyntsev, A. T., Grechnev, V. V., Meshalkina, N. S., \& Yan, Y. 2007, Sol. Phys., 242, 111

Bagenal, F. 1994, J. Geophys. Res., 99, 11043

Barta, M., \& Karlicky, M. 2006, A\&A, 450, 359

Brazhenko, A. I., Bulatsen, V. G., Vashchishin, R. V., et al. 2005, Kinematika i Fizika Nebesnykh Tel Supplement, 5, 43

Carr, T. D., Desch, M. D., \& Alexander, J. K. 1983, in Physics of the Jovian Magnetosphere, ed. A. J. Dessler (New York: Cambridge Univ. Press), 226

Chernov, G. P. 1976, Space Sci. Rev., 20, 582

Chernov, G. P. 2006, Space Sci. Rev., 127, 195

Chernov, G. P. 2010, RA\&A, 10, 821

Dulk, G. A., Lecacheux, A., \& Leblanc, Y. 1992, A\&A, 253, 292

Dulk, G. A., Erickson, W. C., Manning, R., \& Bougeret, J.-L. 2001, A\&A, 365, 294

Genova, F., \& Aubier, M. G. 1985, A\&A, 150, 139

Genova, F., Aubier, M. G., \& Lecacheux, A. 1981, A\&A, 104, 229

Gurnett, D. A., Persoon, A. M., Kurth, W. S., Roux, A., \& Bolton, S. J. 2001, J. Geophys. Res.: Space Phys., 106, 26225

Hankins, T. H., \& Eilek, J. A. 2007, ApJ, 670, 693

Hess, S. L. G., Bonfond, B., Zarka, P., \& Grodent, D. 2011, J. Geophys. Res. (Space Physics), 116, 5217

Hess, S. L. G., Echer, E., Zarka, P., Lamy, L., \& Delamere, P. A. 2014, Planet. Space Sci., 99, 136

Hinson, D. P., Flasar, F. M., Kliore, A. J., et al. 1997, Geophys. Res. Lett., 24, 2107

Hinson, D. P., Twicken, J. D., \& Karayel, E. T. 1998, J. Geophys. Res.: Space Phys., 103, 9505

Imai, K., Wang, L., \& Carr, T. D. 1997, J. Geophys. Res., 102, 7127

Kaiser, M. L. 1993, J. Geophys. Res., 98, 18757

Konovalenko, A., Sodin, L., Zakharenko, V., et al. 2016, Exp. Astron., 42, 11

Konovalenko, A. A. 2000, Washington DC American Geophysical Union Geophysical Monograph Series, 119, 311

Krauss, J. D. 1966, McGraw Hill, New York

Kuijpers, J. 1975, A\&A, 40, 405

Kurth, W. S., Hospodarsky, G. B., Gurnett, D. A., et al. 2001, in Planetary Radio Emissions V, eds. H. O. Rucker, M. L. Kaiser, \& Y. Leblanc, 15

Kuznetsov, A. A., \& Vlasov, V. G. 2013, Planet. Space Sci., 75, 167

Leblanc, Y., Genova, F., \& de La Noe, J. 1980, A\&A, 86, 342

Ledenev, V. G., Yan, Y., \& Fu, Q. 2006, Sol. Phys., 233, 129

Litvinenko, G. V., Rucker, H. O., Vinogradov, V. V., Ryabov, B. P., \& Shaposhnikov, V. E. 2000, Radio Science, 35, 1445

Litvinenko, G. V., Lecacheux, A., Rucker, H. O., et al. 2009, A\&A, 493, 651

Litvinenko, G. V., Shaposhnikov, V. E., Konovalenko, A. A., et al. 2016, Icarus, 272,80

Marques, M. S., Zarka, P., Echer, E., et al. 2017, A\&A, 604, A17

Panchenko, M., Rucker, H. O., Kaiser, M. L., et al. 2010, Geophys. Res. Lett., 37, 5106

Panchenko, M., Rucker, H. O., \& Farrell, W. M. 2013, Planet. Space Sci., 77, 3 Riihimaa, J. J. 1977, Ap\&SS, 51, 363

Riihimaa, J. J. 1979, A\&A, 78, L21

Ryabov, V. B., Vavriv, D. M., Zarka, P., et al. 2010, A\&A, 510, A16

Ryabov, V. B., Zarka, P., Hess, S., et al. 2014, A\&A, 568, A53

Shaposhnikov, V. E., Kocharovsky, V. V., Kocharovsky, V. V., et al. 1997, A\&A, 326, 386

Titova, E. E., Demekhov, A. G., Pasmanik, D. L., et al. 2007, Geophys. Res. Lett., 34, L02112

Treumann, R. A., Nakamura, R., \& Baumjohann, W. 2011, Ann. Geophys., 29, 1673

Zakharenko, V., Konovalenko, A., Zarka, P., et al. 2016, J. Astron. Instrum., 5, 1641010

Zarka, P. 1998, J. Geophys. Res., 103, 20159

Zarka, P., Cecconi, B., \& Kurth, W. S. 2004, J. Geophys. Res. (Space Physics), 109, A09S15

Zheleznyakov, V. V., \& Zlotnik, E. Y. 1975, Sol. Phys., 44, 461

Zheleznyakov, V. V., Zlotnik, E. Y., Zaitsev, V V., \& Shaposhnikov, V. E. 2016, Phys. Usp., 59, 997

Zlotnik, E. Y. 2009, Central European Astrophysical Bulletin, 33, 28

Zlotnik, E. Y., Shaposhnikov, V. E., \& Zaitsev, V. V. 2016, J. Geophys. Res.: Space Phys., 121, 5307 